\title{
ARTIGO ORIGINAL Empatia e reconhecimento de expressões faciais de emoções básicas e complexas em estudantes de Medicina
}

\author{
Empathy and facial expression recognition of basic \\ and complex emotions in medical students
}

Ramon Felix de Ávila', Daiene de Morais², Ana Julia Bomfim², Marcos Hortes N. Chagas²

\section{RESUMO}

Objetivo: Avaliar a empatia e a capacidade de reconhecimento de emoções básicas e complexas e suas correlações em estudantes de Medicina. Métodos: $O$ desenho do estudo foi transversal. Foram avaliados 86 alunos do $3^{\circ}$ e $4^{\circ}$ ano do curso de Medicina de uma faculdade de Medicina do interior do estado de São Paulo com os seguintes instrumentos: (i) escala Jefferson de empatia, (ii) tarefa de Reconhecimento de Expressões Faciais de emoções básicas (REF) e (iii) Reading the mind in the eyes test (RMEt). Resultados: A média geral de acertos no REF foi 15,6 (DP: $\pm 2,3$ ). Houve diferença estatisticamente significante no número de acertos da emoção tristeza no sexo feminino comparado com o masculino $\left(t_{84}=2,30 ; p=0,02\right)$. Em relação ao RMEt, a média geral de acertos foi de 26,5 (DP: $\pm 3,3)$ com diferença estatisticamente significante entre os gêneros com maior número de acertos entre as estudantes do sexo feminino $\left(t_{84}=3,43 ; p<0,01\right)$. O escore total médio na escala de empatia foi 121,3 (DP: $\pm 9,8)$. Houve correlação positiva fraca entre o escore total da escala de empatia e o número de acertos para a emoção tristeza $(r=0,29 ; p<0,01)$. Conclusão: $O$ número de acertos para

\section{Palavras-chave}

Empatia, reconhecimentos de emoções faciais, cognição social, estudantes de Medicina. a emoção tristeza no REF e o escore total do RMEt foi maior no sexo feminino comparado com sexo masculino. Além disso, a empatia parece estar diretamente relacionada com a capacidade de reconhecer a emoção tristeza. Outros estudos parecem pertinentes para avaliar de forma mais profunda aspectos de empatia e reconhecimento de expressões faciais da emoção em estudantes de medicina.

\section{ABSTRACT}

Objective: The aim was to evaluate empathy and facial expression recognition of basic and complex emotions and their correlations in medical students. Methods: A cross-sectional study was performed in a medical school of the state of São Paulo. We evaluated 86 third and fourth year medical students with the following instruments: (i) Jefferson Scale of Empathy, (ii) Facial Expression Recognition of basic emotions task (REF) and (iii) Reading the Mind in the Eyes test (RMEt). Results: The overall mean score in the REF was 15.6 (SD: \pm 2.3 ). There

1 Faculdade de Ciências da Saúde de Barretos Dr. Paulo Prata.

2 Universidade Federal de São Carlos (UFSCar), Centro de Ciências Biológicas e da Saúde.

Recebido em

22/4/2016

Aprovado em

$25 / 7 / 2016$

DOI: $10.1590 / 0047-2085000000126$
Endereço para correspondência: Marcos Hortes N. Chagas

Universidade Federal de São Carlos

Centro de Ciências Biológicas e da Saúde

Rodovia Washington Luís, Km 235

13565-905 - São Carlos, SP, Brasil

Telefone: (55 16) 3306-6938

E-mails: setroh@hotmail.com

mchagas@ufscar.br 


\section{Keywords}

Empathy, facial emotion recognition, social cognition, medical students. was a statistical significant difference in the number of correct responses in sadness emotion in female gender compared to male $\left(t_{84}=2.30 ; p=0.02\right)$. Regarding RMEt, the overall mean score was 26.5 (SD: \pm 3.3 ) with statistically significant difference between genders with higher number of correct responses among female students $\left(t_{84}=3.43 ; p<0.01\right)$. The mean score on the empathy scale was 121.3 (SD: \pm 9.8 ). There was significant weak positive correlation between the total score of empathy scale and the recognition of sadness facial emotion ( $r=$ 0.29; $p<0.01$ ). Conclusion: The number of correct responses to sadness emotion in the REF and the total score of RMEt was higher in female students compared to male. In addition, empathy seems to be directly related to the ability to recognize sadness. Other studies are needed to provide a deeper understanding of empathy and facial emotion recognition in medical students.

\section{INTRODUÇÃO}

As Diretrizes Curriculares Nacionais para graduação no curso de Medicina preconizam que o graduado tenha formação generalista, humanista, crítica e reflexiva'. Dessa forma, o médico será capacitado a atuar pautado em princípios éticos e dimensões humanísticas, desenvolvendo atitudes e valores adequados para uma relação médico-paciente de confiança. Nesse contexto, a empatia com certeza é um dos elementos essenciais para boa relação médico-paciente².

A empatia consiste na habilidade social de compreender o sentimento e a perspectiva do outro ${ }^{3}$. Assim, ela é componente essencial para eficácia terapêutica e está associada à melhora da qualidade de vida dos pacientes, visto que melhores resultados clínicos são encontrados em pacientes cujos médicos se mostravam mais empáticos ${ }^{24}$.

A empatia pode ser dividida em aspectos cognitivos e afetivos, destacando-se no contexto médico e do futuro médico a empatia afetiva 5 . Stepien e Baernstein apontam que a competência médica precisa ir além do processo intelectual, exigindo também habilidade empática ${ }^{6}$. Apesar disso, o desenvolvimento e a manutenção de um comportamento empático adequado no estudante de Medicina podem encontrar barreiras devido aos diversos fatores estressores presentes na graduação ${ }^{7}$. Dentre esses fatores, encontra-se na literatura a sobrecarga de trabalho, a privação do sono e as questões sobre o planejamento da carreira ${ }^{8}$.

A habilidade para reconhecer emoções básicas e complexas no outro é capacidade essencial para a vida em sociedade e está inserida em um domínio cognitivo complexo denominado cognição social ${ }^{9}$. Esse domínio inclui a capacidade de entender as intenções de outra pessoa, de compreender normas, procedimentos e regras sociais ${ }^{9,10}$.

Diversos instrumentos são utilizados para avaliar a cognição social, destacando aqueles que avaliam a teoria da mente e o reconhecimento de expressões faciais de emoções básicas. Premack e Woodruff foram os primeiros pesquisadores a investigar a existência de uma teoria da mente, a qual está relacionada com a habilidade de inferir estados mentais a si e aos outros ${ }^{11}$. Entre os testes de teoria da mente que avaliam aspectos emocionais, destaca-se o Reading the
Mind in the Eyes, que avalia a capacidade de reconhecimento de uma emoção complexa a partir da região dos olhos ${ }^{12}$. Esse instrumento possibilita a avaliação de emoções, além das básicas ${ }^{13}$, tais como apavorado, preocupado, arrependido, chateado, desafiador, hostil, entre outras. As emoções complexas são construídas a partir das emoções básicas e da cultura na qual se insere cada indivíduo ${ }^{14}$.

Em relação ao reconhecimento de expressões faciais de emoções básicas, os primeiros estudos relativos à universalidade das emoções faciais humanas básicas remontam à publicação de Darwin no ano de 1872 intitulada "A expressão das emoções no homem e nos animais"15. Atualmente, o caráter universal das emoções básicas e sua importância na interação social são reconhecidos, propondo-se a existência de seis emoções básicas: tristeza, alegria, nojo, medo, raiva e surpresa ${ }^{16}$. Estudos com diferentes paradigmas são utilizados para avaliar o reconhecimento de expressões faciais de emoções básicas ${ }^{17,18}$ e a maioria utiliza as faces de Ekman e Friesen ${ }^{16}$, também empregadas neste estudo.

É interessante notar que tanto os níveis de empatia como a capacidade de reconhecimento de expressões faciais das emoções parecem ser mais altos em mulheres comparados com homens, independentemente da amostra estudada. Uma revisão sistemática recente avaliou 26 estudos, dos quais 16 apontaram para um melhor desempenho das mulheres no reconhecimento de expressões faciais ${ }^{19}$. No mesmo sentido, Santos et al. encontraram níveis de empatia maiores em estudantes de Medicina do sexo feminino comparado com o sexo masculino ${ }^{20}$.

Apesar da importância do reconhecimento de expressões faciais e empatia em estudantes de Medicina, nenhum estudo foi realizado até o momento para a avaliar a correlação entre essas variáveis. A hipótese deste estudo é de que estudantes com maiores níveis de empatia apresentam maior capacidade de reconhecer emoções faciais. Dessa forma, este estudo tem como objetivo avaliar a empatia e a capacidade de reconhecimento de emoções básicas e complexas e suas correlações em estudantes de medicina do $3^{\circ}$ e $4^{\circ}$ anos de uma faculdade do interior do estado de São Paulo. 


\section{MÉTODOS}

\section{Local e participantes}

O estudo foi realizado em uma faculdade particular do interior do estado de São Paulo, com alunos do curso de Medicina. O curso tem entrada anual de 60 alunos e a faculdade foi implantada em 2012. Dessa forma, os participantes deste estudo são alunos da primeira e segunda turma da faculdade. Outra característica importante é que a faculdade possui apenas o curso de graduação em Medicina.

Neste estudo, foram avaliados todos os alunos do $3^{\circ}$ e $4^{\circ}$ anos, totalizando 86 alunos, o que corresponde a $74 \%$ dos alunos matriculados no ciclo clínico. Os demais estudantes não aceitaram participar do estudo. Neste estudo, foi definido avaliar apenas alunos do ciclo clínico pelo maior contato com os pacientes comparado com o ciclo básico ( $1^{\circ}$ e $2^{\circ}$ anos).

\section{Instrumentos}

Os seguintes instrumentos foram utilizados:

\section{Reconhecimento de Expressões Faciais de emoções básicas (REF)}

Esta tarefa foi feita e apresentada com a utilização do software Superlab 4.0 (Cedrus corporation ${ }^{\circledR}$ ) em um computador com tela de 13 polegadas da marca Dell ${ }^{\circledR}$ (modelo 7348). A tarefa consistiu na apresentação de 28 imagens, sendo as duas primeiras para treinamento do participante. Durante o teste, imagens de faces de dois atores (um homem e uma mulher) eram apresentadas de forma aleatória, sendo 24 de emoções básicas (alegria, tristeza, medo, nojo, raiva e surpresa) nas intensidades de $30 \%$ e $70 \%$ e duas faces neutras. A escolha dessas intensidades emocionais foi baseada em outros estudos que utilizaram paradigmas semelhantes ${ }^{21,22}$. As fotografias, todas nas cores branco e preto, foram retiradas do banco de estímulos criado e validado por Ekman e Friesen ${ }^{16}$. O participante teve cinco segundos para observar a imagem e então deveria escolher entre sete opções que apareciam na tela (alegria, tristeza, medo, nojo, raiva, surpresa, neutro). Para escolha da opção, o participante utilizava o mouse. Não existia tempo limite para responder, de forma que o participante passa para a próxima imagem automaticamente ao responder a anterior.

\section{Reading the Mind in the Eyes test (RMEt)}

Este teste avalia a habilidade de compreender as intenções, os sentimentos e os pensamentos de outra pessoa por meio de suas expressões faciais, especialmente na região dos olhos $^{12}$. Foi aplicada a versão computadorizada do RMEt. O teste contém 36 figuras de emoções complexas da região da face do meio do nariz até pouco acima da sobrancelha, todas em preto e branco. Cada imagem está cercada por quatro palavras representando estados mentais. Os participantes devem escolher a palavra que melhor representa o estado mental apresentado. O resultado do teste foi avaliado em termos de pontuação total de acertos. Esse teste foi recentemente validado e adaptado para o Brasil ${ }^{23}$.

\section{Escala Jefferson de Empatia médica (versão para estudantes)}

Esta escala autoaplicada apresenta 20 itens, divididos em itens positivos e negativos. Os itens positivos variam de 1 (discorda fortemente) a 7 (concorda fortemente) e os itens negativos de forma inversa de 1 (concorda fortemente) a 7 (discorda fortemente). Dessa forma, o escore total da escala varia de 20 a 140 com maiores escores significando maior empatia ${ }^{24}$. A escala é composta por três domínios: tomada de perspectiva (10 itens), compaixão (8 itens), e a capacidade de se colocar no lugar do paciente ( 2 itens) $)^{24}$. A versão brasileira da escala foi validada por meio de um estudo realizado com 319 estudantes de medicina da Universidade de São Paulo25.

\section{Procedimentos}

O aluno foi convidado a participar das avaliações de reconhecimento de expressões faciais e empatia. Esse convite foi feito pelos pesquisadores e, assim que o aluno concordou em participar, foi combinado um horário apropriado para a coleta de dados. As coletas foram realizadas de forma individual no campus da faculdade, em sala de aula, após o término desta, buscando a garantia de um ambiente adequado e tranquilo.

O projeto foi aprovado no Comitê de Ética em Pesquisa (CAAE: 39732814.0.0000.5378, parecer: 959.395) e todos os participantes assinaram o termo de consentimento livre e esclarecido.

\section{Análise de dados}

Os dados sociodemográficos foram analisados por meio do teste $t$ de Student para dados contínuos com distribuição normal, teste não paramétrico de Mann-Whitney para dados contínuos sem distribuição normal e pelo qui-quadrado $\left(X^{2}\right)$ para dados nominais. Para variáveis quantitativas, foram calculadas média, desvio-padrão (DP) e erro-padrão da média (EP). As correlações entre o número de acertos (total e por emoção) das tarefas de reconhecimento de expressões faciais das emoções (REF e RMEt) e o escore total da escala de empatia foram analisadas pelos coeficientes de correlação de Pearson (dados com distribuição normal) e Spearman (dados sem distribuição normal). A distribuição da amostra foi avaliada pelo teste de Kolmogorov-Smirnov.

Os dados foram analisados usando o programa SPSS-21 e o nível de significância adotado foi $p<0,05$.

\section{RESULTADOS}

Em relação às características da amostra, a idade média foi $22,4$ anos (DP: $\pm 2,09 ;$ EP: $\pm 0,23), 75,6 \%(n=65)$ da amostra 
era do sexo feminino, $97,7 \%$ dos estudantes eram solteiros e 96,5\% declararam ser brancos.

Considerando os escores do REF, a média de acertos totais foi 15,6 (DP: $\pm 2,25$; EP: $\pm 0,24$ ) com mínimo de 10 acertos e máximo de 20 acertos. A média do número de acertos totais para emoções em intensidade de $70 \%$ foi 10,1 (DP: \pm 1,40; EP: $\pm 0,15$ ) [min: 7; máx: 12] e para emoções em intensidade de 30\% foi 3,9 (DP: $\pm 1,53$; EP: $\pm 0,16$ ) [min: 1; máx: 7]. A Tabela 1 mostra a porcentagem de participantes de acordo com o número de acertos em cada emoção no REF.

Em relação ao sexo, houve diferença estatisticamente significante no número de acertos da emoção tristeza no sexo feminino comparado com o masculino $\left(t_{84}=2,30 ; p=0,02\right)$. Não houve diferenças para o escore total e demais emoções.

No RMEt, a média de acertos totais foi 26,5 (DP: $\pm 3,32$; EP: $\pm 0,36)$ com escore mínimo de 18 e máximo de 33. Neste instrumento, o sexo feminino também apresentou escore mais elevado comparado com o sexo masculino $\left(t_{84}=3,43\right.$; $\mathrm{p}<0,01)$.

O escore total médio na escala Jefferson de empatia foi 121,3 (DP: $\pm 9,84 ;$ EP: $\pm 1,06$ ) com pontuação mínima de 78 e máxima de 140. Não houve relação entre empatia e dados sociodemográficos avaliados.

Em relação às correlações entre a empatia e os instrumentos REF e RMEt, os escores de empatia apresentaram correlação positiva fraca com o reconhecimento da emoção tristeza na tarefa REF $(r=0,29 ; p<0,01)$. Não houve correlação com as demais emoções e com o RMEt.

Tabela 1. Porcentagem de participantes de acordo com o número de acertos no Teste de Reconhecimento de Expressões Faciais de Emoções Básicas divididos por emoções

\begin{tabular}{cccccccc}
\hline $\begin{array}{c}\text { Número } \\
\text { de } \\
\text { acertos }\end{array}$ & Alegria & Tristeza & Raiva & Nojo & Surpresa & Medo & Neutro \\
\hline 0 & $0 \%$ & $0 \%$ & $0 \%$ & $2,3 \%$ & $0 \%$ & $7 \%$ & $7 \%$ \\
1 & $22,1 \%$ & $24,4 \%$ & $2,3 \%$ & $14 \%$ & $3,5 \%$ & $16,3 \%$ & $24,4 \%$ \\
2 & $51,2 \%$ & $43 \%$ & $23,3 \%$ & $60,5 \%$ & $29,1 \%$ & $40,7 \%$ & $68,6 \%$ \\
3 & $26,7 \%$ & $31,4 \%$ & $54,7 \%$ & $20,9 \%$ & $54,7 \%$ & $31,4 \%$ & - \\
4 & $0 \%$ & $1,2 \%$ & $19,8 \%$ & $2,3 \%$ & $12,8 \%$ & $4,7 \%$ & - \\
\hline
\end{tabular}

\section{DISCUSSÃO}

Entre os resultados, os seguintes aspectos devem ser destacados: a capacidade de reconhecer a emoção básica tristeza e emoções complexas foi maior no sexo feminino, comparado com o masculino em nossa amostra. Além disso, houve correlação positiva fraca entre a empatia e a capacidade de reconhecer a emoção tristeza.

O escore médio de empatia encontrado em nossa amostra foi maior do que em relação a outros estudos que utilizaram a mesma escala. No estudo de validação do instrumento no Brasil, a média na escala Jefferson de empatia foi 114,9
(DP: $\pm 12,4)^{25}$. Da mesma forma, um estudo ${ }^{26}$ realizado com estudantes japoneses encontrou escore médio de 104,3 (DP: $\pm 13,1)$ e outro ${ }^{27}$ com estudantes do Reino Unido e da Nova Zelândia encontrou escore de 111,4 (DP: $\pm 12,0$ ). Essas diferenças entre os estudos podem estar relacionadas a características da amostra, como gênero, ano de formação do estudante e características curriculares do curso. Por exemplo, escolas médicas com grade curricular com maior número de disciplinas com formação humanística ou com mais estudantes do sexo feminino poderiam se relacionar com maiores escores de empatia. Na faculdade estudada, deve-se destacar a presença de disciplinas humanísticas desde o primeiro ano do curso e a ligação extremamente próxima com um hospital filantrópico para o tratamento de câncer.

Todavia, deve-se destacar também que diversos estudos realizados em estudantes de Medicina apontam para um declínio significativo na empatia durante a graduação ${ }^{28-31}$, o que reforça ainda mais a necessidade de disciplinas humanísticas na grade curricular do curso de Medicina. Riess et al. testaram um protocolo de treinamento de empatia em médicos-residentes e encontraram melhora nos escores de empatia e aumento na habilidade de reconhecer emoções faciais ${ }^{32}$.

Apesar de a correlação entre empatia e o reconhecimento da emoção tristeza ter sido fraca, esse achado pode revelar processos cognitivos envolvidos nas diferenças de níveis de empatia encontrados entre as pessoas. Considerando que a empatia pode ser definida como a capacidade de se colocar na perspectiva do outro ${ }^{3}$, esse processo passa primeiramente pela habilidade de reconhecer a emoção presente no outro seja por estímulos verbais como não verbais 9 . Dessa forma, o reconhecimento de estado emocional a partir da face poderia ser um dos pontos de partida para a habilidade empática. De maneira semelhante, um estudo que avaliou a relação entre o RMEt e a empatia demonstrou correlação fraca entre os escores ${ }^{33}$, reforçando uma possível relação entre empatia e reconhecimento de emoções faciais. Considerando apenas a correlação entre tristeza e empatia, vale lembrar que o mecanismo de empatia é muitas vezes relacionado com reconhecimento de presença de dor no outro, possivelmente devido a processos evolutivos ${ }^{34}$. Esses achados podem ser extrapolados para o achado do estudo considerando a relação entre dor e tristeza.

Em relação à maior porcentagem de acerto de reconhecimento de emoções faciais por estudantes do sexo feminino, outros estudos apontaram na mesma direção e encontraram maior capacidade das mulheres em reconhecer emoções faciais independente da amostra utilizada ${ }^{19}$. Dessa forma, esse achado pode não ter relação direta especificamente com a amostra de estudantes de Medicina. A hipótese para o melhor desempenho do sexo feminino está associada aos aspectos culturais e evolutivos. A atribuição de cuidar de uma criança, especialmente na fase pré-verbal do desenvol- 
vimento, pode auxiliar no desenvolvimento de habilidades necessárias para o reconhecimento de emoções ${ }^{19,35}$.

Considerando os achados mencionados, fica clara a necessidade de mais estudos que explorem a empatia e o reconhecimento de expressões faciais das emoções em estudantes de Medicina. Assim, devem-se apontar as limitações deste estudo que incluem o desenho de estudo transversal utilizando apenas alunos do ciclo clínico ( $3^{\circ}$ e $4^{\circ}$ anos) e a amostra pequena. Outros estudos poderiam incluir todos os anos da formação, apresentar desenho longitudinal e comparar grades curriculares das diferentes escolas médicas. Além disso, poder-se-ia incluir instrumentos de reconhecimento de emoções com paradigmas diferentes de preferência com maior número de imagens por emoção básica. Futuros estudos também deverão considerar aspectos como medidas de inteligência dos respondentes e presença de transtornos psiquiátricos, considerando suas influências na cognição social. Por fim, deve-se salientar que as tarefas foram realizadas independentemente do período do ciclo menstrual das respondentes femininas e sabe-se que esse fator pode interferir na acurácia de tarefas de reconhecimento das expressões faciais ${ }^{36}$.

\section{CONCLUSÕES}

O número de acertos para a emoção tristeza no REF e o escore total do RMEt foram maiores no sexo feminino comparado com sexo masculino, o que apenas confirmou um achado da literatura, que aponta para esse resultado independente da amostra. Além disso, os escores de empatia foram diretamente relacionados com o reconhecimento da emoção básica tristeza. Apesar de essa correlação ser fraca, ela pode indicar que o reconhecimento e o processamento cognitivo da emoção facial do outro podem estar relacionados com a capacidade empática. Estudos que avaliem o impacto de disciplinas de formação humanística nos níveis de empatia seriam desejáveis, assim como os que abordem mais aspectos da relação analisada nesta pesquisa, visto a importância destes na formação médica.

\section{CONTRIBUIÇÕES INDIVIDUAIS}

Ramon Félix de Ávila - Elaboração da estrutura do trabaIho, aplicação dos testes, avaliação dos resultados, primeira redação do artigo.

Daiene de Morais - Elaboração da estrutura do trabaIho, correção e redação final do artigo.

Ana Julia Bomfim - Elaboração da estrutura do trabaIho, correção e redação final do artigo.

Marcos Hortes Nisihara Chagas - Elaboração da estrutura do trabalho, análise estatística dos dados, correção e redação final do artigo.

\section{CONFLITOS DE INTERESSE}

Os autores declararam não haver conflitos de interesse.

\section{AGRADECIMENTOS}

Agradecemos ao Centro de Bioética do Conselho Regional de Medicina do Estado de São Paulo (Cremesp) pelo financiamento da pesquisa.

\section{REFERÊNCIAS}

1. Brasil. Ministério da Educação. Conselho Nacional de Educação. Câmara de Educação Superior. Resolução CNE/CES 3/3014. Institui diretrizes curriculares nacionais do curso de graduação em medicina. Diário Oficial da União. Brasília, 23 jun. 2014; Seção 1. p. 8-11.

2. Halpern J. What is clinical empathy? J Gen Intern Med. 2003;18(8):670-4.

3. Melloni M, Lopez V, Ibanez A. Empathy and contextual social cognition. Cogn Affect Behav Neurosci. 2014;14(1):407-25

4. Stewart MA. Effective physician-patient communication and health outcomes: a review. CMAJ. 1995;152(9):1423-33.

5. Hojat M, Gonnella JS, Mangione S, Nasca TJ, Veloski JJ, Erdmann JB, et al. Empathy in medical students as related to academic performance, clinical competence and gender. Med Educ. 2002;36(6):522-7.

6. Stepien KA, Baernstein A. Educating for empathy. A review. J Gen Intern Med. 2006;21(5):524-30

7. Park KH, Kim D, Kim SK, Yi YH, Jeong JH, Chae J, et al. The relationships between empathy, stress and social support among medical students. Int J Med Educ. 2015;6:103-8.

8. Levey RE. Sources of stress for residents and recommendations for programs to assist them. Acad Med. 2001;76(2):142-50.

9. Mitchell RLC, Phillips LH. The overlapping relationship between emotion perception and theory of mind. Neuropsychologia. 2015;70:1-10

10. American Psychiatric Association. Diagnostic and Statistical Manual of Mental Disorders. 5th ed. Washington DC: American Psychiatric Press; 2013.

11. Premack D, Woodruff $G$. Does the chimpanzee have a theory of mind? Behav Brain Sci. 1978:1(4):515-26

12. Baron-Cohen S, Wheelwright S, Hill J, Raste Y, Plumb I. The "Reading the Mind in the Eyes" Test revised version: a study with normal adults, and adults with Asperger syndrome or high-functioning autism. J Child Psychol Psychiatry. 2001;42(2):241-51.

13. Maurage P, Grynberg D, Noël X, Joassin F, Hanak C, Verbanck P, et al. The "Reading the Mind in the Eyes" test as a new way to explore complex emotions decoding in alcohol dependence. Psychiatry Res. 2011;190(2-3):375-8.

14. Ekman P, Sorenson ER, Friesen WV. Pan-cultural elements in facial displays of emotion. Science. 1969;164(3875):86-8.

15. Darwin C. A expressão das emoções no homem e nos animais. São Paulo: Companhia das Letras; 2009.

16. Ekman P, Friesen W. Pictures of Facial Affect. Palo Alto: Consulting Psychologists; 1976

17. Machado-de-Sousa JP, Arrais KC, Alves NT, Chagas MH, de Meneses-Gaya C, Crippa JA, et al. Facial affect processing in social anxiety: tasks and stimuli. J Neurosci Methods. 2010;193(1):1-6

18. da Silva Ferreira GC, Crippa JAS, de Lima Osório F. Facial emotion processing and recognition among maltreated children: a systematic literature review. Front Psychol. 2014;5:1460.

19. Forni-Santos L, Osório FL. Influence of gender in the recognition of basic facial expressions: a critical literature review. World J Psychiatry. 2015;5(3):342-51.

20. Santos MA, Grosseman S, Morelli TC, Giuliano ICB, Erdmann TR. Empathy differences by gender and specialty preference in medical students: a study in Brazil. Int J Med Educ. 2016;7:149-53 
21. Kornreich C, Blairy S, Philippot P, Dan B, Foisy M, Hess U, et al. Impaired emotional facial expression recognition in alcoholism compared with obsessive-compulsive disorder and normal controls. Psychiatry Res. 2001;102(3):235-48.

22. Foisy ML, Kornreich C, Fobe A, D'Hondt L, Pelc I, Hanak C, et al. Impaired emotional facial expression recognition in alcohol dependence: do these deficits persist with midterm abstinence? Alcohol Clin Exp Res. 2007;31(3):404-10.

23. Sanvicente-Vieira B, Kluwe-Schiavon B, Wearick-Silva LE, Piccoli GL, Scherer L, Tonelli $H A$, et al. Revised Reading the Mind in the Eyes Test (RMET) - Brazilian version. Rev Bras Psiquiatr. 2014;36(1):60-7.

24. Hojat M, Mangione S, Nasca TJ, Cohen MJ, Gonnella JS, Erdmann JB, et al. The Jefferson Scale of Physician Empathy: Development and Preliminary Psychometric Data. Educ Psychol Meas. 2001;61(2):349-65.

25. Paro HBMS, Daud-Gallotti RM, Tibério IC, Pinto RMC, Martins MA. Brazilian version of the Jefferson Scale of Empathy: psychometric properties and factor analysis. BMC Med Educ. 2012;12(1):73.

26. Kataoka HU, Koide N, Ochi K, Hojat M. Measurement of empathy among Japanese medical students: psychometrics and score differences by gender and level of medical education. Acad Med. 2009;84(9):1192-7.

27. Quince TA, Kinnersley P, Hales J, da Silva A, Moriarty H, Thiemann P, et al. Empathy among undergraduate medical students: a multi-centre cross-sectional comparison ofstudents beginning and approaching the end of their course. BMC Med Educ. 2016;16(1):92.
28. Chen D, Lew R, Hershman W, Orlander J. A cross-sectional measurement of medical student empathy. J Gen Intern Med. 2007;22(10):1434-8.

29. Hojat M, Mangione S, Nasca TJ, Rattner S, Erdmann JB, Gonnella JS, et al. An empirical study of decline in empathy in medical school. Med Educ. 2004;38(9):934-41.

30. Hojat M, Vergare MJ, Maxwell K, Rattner S, Erdmann JB, Gonnella JS, et al. The devil is in the third year: a longitudinal study of erosion of empathy in medical school. Acad Med. 2009;84(9):1182-91.

31. Neumann M, Edelhäuser F, Tauschel D, Fischer MR, Wirtz M, Woopen C, et al. Empathy decline and its reasons: a systematic review of studies with medical students and residents. Acad Med. 2011;86(8):996-1009.

32. Riess H, Kelley JM, Bailey RW, Dunn EJ, Phillips M. Empathy training for resident physicians: A randomized controlled trial of a neuroscience-informed curriculum. J Gen Intern Med. 2012;27(10):1280-6.

33. Youssef FF, Nunes P, Sa B, Williams S. An exploration of changes in cognitive and emotional empathy among medical students in the Caribbean. Int J Med Educ. 2014;5:185-92.

34. Danziger N, Prkachin KM, Willer JC. Is pain the price of empathy? The perception of others' pain in patients with congenital insensitivity to pain. Brain. 2006;129(Pt 9):2494-507.

35. Kret ME, De Gelder B. A review on sex differences in processing emotional signals. Neuropsychologia. 2012;50(7):1211-21.

36. Derntl B, Kryspin-Exner I, Fernbach E, Moser E, Habel U. Emotion recognition accuracy in healthy young females is associated with cycle phase. Horm Behav. 2008;53(1):90-5. 